\title{
Meetings bloody meetings: a transition in the concept of meeting as technology reduces dependency on time and geography
}

\author{
$W \mathrm{G}$ Hewett \\ School of MIS (Warrnambool Campus), Faculty of Management, Deakin \\ University, PO Box 423 Warrnambool Victoria 3280, Australia, telephone \\ 6155 633228,fax 6155 633320, email billh@deakin.edu.au
}

\begin{abstract}
In geographically spread organisations the cost of meetings can be significantly higher than it is in single location enterprises. The use of technology to support meetings simultaneously held in multiple locations has been promoted as a potential solution. Traditional cost/benefit analysis used in justifying the implementation of this technology shows that success should be assured. The time lost in travel is a visible and easily measured cost of running a meeting, savings in travel (absolute travel and accommodation costs together with notional time lost costs) can be used to justify significant investments in meeting support technology.

There are, however, many problems in successfully changing an organisation's meeting culture so that it freely embraces the use of technology. Many organisations have difficulty in the implementation and use of meeting support technologies. Further, after the initial flush of enthusiasm, facilities are often poorly utilised resulting in significant lost opportunities. There are less tangible costs of corporate meetings which are often more significant than the easily measured savings from travel avoided. The identification of these costs might aid the longer term acceptance of meeting support technology.

Key among the problems to be addressed is the difficulty some meeting chairs have in successfully running a meeting, spread over several locations and supported by technology such as video and audio facilities. These difficulties can be traced to poor pre-meeting preparation and poor understanding of the technologies chosen. They are exacerbated by the failure of participants to understand the true nature of the potential savings available. The result is often failed projects or poorly utilised facilities.

It is with this in mind that this paper has been developed. Only some of the "Pluses and Minuses" in the use of video and audio meeting technology are addressed, along with some of the issues that could be used to develop a "briefing to participants paper" for use in minimising some identified problems in the use of the technology in support of formal meetings. Finally, several unanswered questions are posed. Questions which should be subjected to further study and analysis.
\end{abstract}

Keywords
Information Technology, Video Conferencing, Meeting Support 


\section{STRUCTURE OF THE PAPER}

Two general case studies have been used to enable the identification of the opportunities, costs and issues involved in meetings held by geographically spread organisations. The first helps support the case that the cost of participants travelling to meetings is significantly higher than a simple calculation of travel time might indicate. The second helps identify a number of issues which emerge when technology is implemented to support multiple location meetings. These cultural and political issues may prove to be the limiting factor in the use of technology to support geographically spread organisations.

The body of the paper addresses proposals to overcome some of the impediments to the successful implementation of meeting support technology. The final section of the paper addresses several issues which emerge as needing further analysis before clear guidelines in justifying and implementing technology to support multiple location meetings.

\section{MEETING TYPES AND DESIRED OUTCOMES}

It is simplistic in the extreme to imagine there is a single class of meeting. Similarly it is simplistic to imagine that technology can equally support all meeting classes. The following breakdown is offered as a simple, but by no means exhaustive, classification. This is achieved using the frequency of the meeting, its desired outcomes and, to a lesser extent, the type of person attending as a the classification mechanism.

See the bibliography for reference to further reading on meetings and organisations. (Renton, 1994; Shockley-Zalabak, 1991)

\subsection{Regular meetings}

The following classification of meeting types roughly defines the forms of meetings typically held:

Regular meetings with formal schedule, clear chairperson, and pre-published agenda.

Regular, but, informal meetings with no fixed agenda but clear chairperson.

Regular informal meetings with no fixed agenda and no clear chairperson.

Ad hoc meetings called to address a particular issue with a clear chairperson, and pre-determined agenda.

Ad hoc meetings for general information exchange with no fixed agenda but clear chairperson.

\subsection{Spontaneous meetings}

Spontaneous meetings, "hallway" meetings or "stand up" meetings are rarely able to be supported by the meeting technology under review here. The expansion of computer mediated communication using groupware ${ }^{1}$, however, is having an impact in the area of spontaneous group decision making.

\subsection{Inter versus intra organisational meetings}

The scope of this paper is limited to observations addressing inter organisational meetings.

However, in both cases reported here, ad hoc meetings were also documented involving members from across organisational boundaries. It is clear from this involvement that simple technology can be used to facilitate intra organisational communication also. One such involved academics from the Faculty of Management, discussing approaches to the introduction of computer mediated

\footnotetext{
1 Groupware is defined as a package which may include document management, e-mail, desktop conferencing, scheduling or electronic-forms applications. The groupware market, dominated by Lotus Notes, was evaluated at US\$ 1 billion in 1994; it is expected to reach US\$ 4 billion by 1998. Computer 28 (9) Sept. 1995.
} 
communication to support the teaching and learning process, with the chief executive of an IT service company. This company decided to involve experienced people from Sydney and Los Angeles in the discussion. This extremely productive meeting was arranged with a minimum of fuss involving facsimile and audio links.

\subsection{Expected outcomes}

The following classification of desired meeting outcome, or just why meetings are called, could be expected:

To deliver communication.

To deliver communication and seek feedback.

To finalise a decision on a single issue.

To finalise a decision on a number of related issues.

To fulfil a regular meeting schedule commitment of a committee or board.

\subsection{People attending meetings}

The following classification of people could be expected at meetings.

Committee or board members.

Project team members, eg. people working on a specific problem.

Department members, eg. people involved in the review of department performance against its budgeted goals.

Members from across organisational divisions, eg. people from Marketing and Manufuctiring reviewing plans for streamlined product delivery.

\section{CASE ONE}

This analysis was of a widely spread manufacturing and retailing organisation. At the time of the analysis it was small to medium in size (\$AUS70-80 million in annual sales) and therefore had limited resources to devote to information technology. This organisation differed from most small organisations in a number of ways. For the purpose of this discussion the most significant differences were:

It was a fully vertically integrated manufacturing and retailing organisation.

It was represented throughout Australia.

The company's head office was located in a regional city (some three hours drive from

Melbourne) along with its major manufacturing plant.

The company's next most significant corporate presence, its marketing office, was in Melbourne.

The company had significant links with raw material and technology suppliers in both the USA and the UK.

\subsection{Technology to support expansion}

In 1976 the company was in the middle of a significant expansion. Sales were increasing at a steady rate. Manufacturing capacity needed to expand to meet the demand. Management involvement was required to streamline this expansion. The company had recognised the need to improve its administration and manufacturing systems and had embarked on a radical technology upgrade path. Computer Aided Design and Manufacturing (CAD/CAM) applications were installed. Traditional MIS applications were developed to support management. point of sale (POS) equipment was installed in shops through out Australia to gather sales details. Portable data entry (PDE) equipment and bar code based tracking systems were implemented. Communications networks were implemented to support 
the POS and PDE data gathering. The company had not recognised, however, the emergence of a significant inter-organisational communication need.

\subsection{Internal communications - the limiting factor}

This need for heightened inter-organisational communications peaked in the early 1980's and was addressed in a number of ways:

Key executives were included in the monthly board meetings.

Regular meetings involving staff in all of the functional divisions were established.

Annual "management" meetings were formalised

Annual Strategic Planning meetings were held followed by monthly executive meetings

examining the company's progress.

Management Information Systems supported by electronic communication networks were introduced.

Electronic mail was introduced, starting at the top and moving down through the organisational layers.

Retail, Manufacturing, and Supply divisions met regularly, supported by electronic decision support and information analysis technology.

\subsection{The problem of distance}

Inevitably, because of the distances involved, key executives were called upon to travel extensively. This was not seen as a significant imposition since the corporate culture had always involved significant travel. The company grew from an initial travelling salesman's decision to put down roots and build a retail organisation. This organisation grew into a manufacturing/retail organisation when the owner decided a higher level of quality was required. The aim was to supply merchandise at a level of quality and price points not available through the regular suppliers. This approach proved successful in the eyes of the consumers and the company grew into an Australia wide organisation in the 1960's and 1970's.

\subsection{The acceptance of travel}

The culture of travel was accepted as the norm by all members of the board and the executive group. Travel was seen as good, because it opened opportunities for key personnel to meet customers and employees and to see just what the opposition was doing. What occurred as the need for more and more communications oriented meetings arose, however, was that key executives spent more time travelling without gaining the traditionally accepted benefits of travel. Less and less of their scarce time was spent actually exposed to customers and the marketplace. More importantly, executives spent less time with retail staff, the organisation's interfaces to the market, while at the same time the costs associated with travel increased.

\subsection{The acceptance of electronic mail}

The electronic mail network, the implementation of which commenced in 1982, was relatively well accepted. In fact the Marketing Director was prompted to comment that the introduction of email had enabled him to "win arguments with the Managing Director he would have lost on the telephone". The reason given was that the asynchronous nature of the email interchange meant that more reasoned and less emotive arguments could be used. The email network was expanded (in a top down fashion) on the back of the communications network installed to deliver traditional manufacturing and retail MIS systems. 


\subsection{The costs}

Increasingly, key executives and managers were participating in meetings held in locations up to three hours from their home base. Many half day and full day strategy and review meetings were held in closed rooms, which could have been anywhere. Because of the time involved in meetings and travel there was little time left for customer and employee interaction.

Thus, the cost of these meetings can be seen as more than travel and accommodation expenses alone. More than the cost of hours lost in executive travel. The indirect cost was a significant loss of market focus. As busy executives attempted to meet more often to address rapid changes in the corporate environment, less and less time was spent involved in the market place.

This loss of market focus is a significant reason for this otherwise technological innovative and outward looking organisation failing to survive the turbulence of the late 1980's and early 1990's. It was found that the company was not well positioned to meet the twin threats of cheap imported goods, as the tariff regime in Australia altered, and a shift in consumer desires towards less durable goods [Hewett \& Symons, 1992].

\subsection{The important issues from case one}

The cost of travel to meetings in an integrated but geographically spread organisation can be significantly higher than the simple addition of the time involved in travel. By only concentrating on travel as a possible cost saving, organisations can overlook the less quantifiable benefits of implementing multi-location meeting technology. The loss of focus and the lost opportunities for wider interaction should not be ignored. Better use of multi-location meeting support technology which was beginning to become available in the late 1980's, when this organisation most needed help, may have enabled the required communication and planning meetings to proceed without the loss of marketplace interaction.

The electronic mail network, while well accepted, is a one to one form of communication support and does not address the one to many and the many to many communication needs which also exist. The introduction of group decision support technology as an extension to the electronic mail network would have been a significant enhancement. Unfortunately GDSS was not readily available at the time.

\section{CASE TWO}

This analysis was of an integrated but geographically spread University with six campus locations in regional and metropolitan Victoria. Three campuses are located in Melbourne's eastern suburbs (in normal traffic about a half an hour's drive between each), the others are located in regional Victoria (from one to three hours drive from Melbourne). The term integrated is chosen here to differentiate this University from similar, geographically spread institutions which have opted for a federated model.

This institution emerged in 1990/91 as a result of a series of mergers. The Australian Government facilitated these mergers in an attempt to rationalise the tertiary education sector. As a result of two closely timed mergers the University is now a relatively large institution, 26000 undergraduate and postgraduate students and an additional 20000 students undertaking professional extension courses through out Australia. It also has a significant number of international students both in Australia and studying abroad.

\subsection{Technology to support integration}

On the administration and academic fronts, the need for change was clear as was the need to involve people from the geographically spread parts of the new entity in new administration and course delivery structures. An early priority was the establishment of electronic communications links between the campuses. One time capital support was used to implement communication links for voice, video and data traffic. Local area networks were put in place and a significant investment was 
made in desktop technology. A strategic investment was also made in video supported conference rooms. These video rooms were initially thought of, and justified, as multi purpose aimed at both administrative meeting support and academic teaching support. Of a lower priority, but in hindsight possibly just as critical to the success of the merger, was the establishment of informal "voice point" meeting rooms.

During the merging of two, and then three, individual business faculties into a single, integrated, Faculty of Management many meetings were held. Many of these meetings were face to face but a significant number used the video facilities installed in the first days of the merged institution. The technology available in 1990/91 was crude when compared to what is possible today. It did function, however, and this technology enabled many interchanges which otherwise would have been impossible.

\subsection{Technology supported ad hoc meetings}

As the merger "politics" became consolidated, less and less use of the video facilities was made for ad hoc meetings. This was possibly because fewer of these meetings were taking place, but the emergence of the informal voice point network was more likely the reason. The purchase of voice point equipment was seen as an "expense" issue and therefore able to be justified at a relatively low corporate level. As people came to know each other, the need for voice to be supported by picture became less and less. The ease by which a voice point meeting could be arranged far outweighed the "bureaucratic booking procedures" required for the video facilities. Further the integrated communications network delivered "unlimited" voice lines to all university locations.

\subsection{Technology supported regular meetings}

Regular meetings persisted over the video facilities. In particular, meetings conducted on a formal schedule, with a clear chairperson, and running to a pre-published agenda are still regularly held using the video facilities. There has been a gradual reduction in the use of video for other formal meetings. This can probably be traced to the consolidation of power in a lesser number of sites. In several instances decisions to call face to face meetings at short notice were seen as deliberate political tactics used to marginalise opponents.

\subsection{Reduction in the use of technology supported meetings}

The reduced use of the technology can also be traced to the failure of the institution to propagate the video meeting room facilities as campus real estate became scarce during a phase of "geographic" reorganisation and consolidation. The need to move to an unfamiliar building some distance from the regular offices of those attending is often cited as a reason why the meeting could not be scheduled using the technology, but instead must be face to face.

The final reason for the reduced use of the video technology is the difficulty some meeting chairs have in successfully running a video meeting. This last reason is seldom explicitly stated, but often emerges as a resistance by those attending, with the often stated claim that video meetings are just not productive. Informal discussions with both experienced and inexperienced meeting chairs support this contention. Further, discussions with those attending video meetings and with those who fail to attend voluntary video supported meetings also support this conclusion.

\subsection{The important issues from case t wo}

The failure to enhance and support the facilities can in itself be seen as an indirect message, from 'corporate management', that the meeting support technology was of only marginal use. Poor meeting outcomes have also been a contributing factor in the reduction of use of the technology. These poor meeting outcomes can be directly linked to the difficulties meeting chairs have in scheduling, running and, controlling multi-location technology supported meetings. 
Thus it can be seen that, even after an investment in meeting support technology has been made, a change in corporate culture is still required in order to ensure the full potential of the technology is delivered.

\section{THE SOLUTION: PRE-MEETING PREPARATION}

Pre-meeting preparation can be broken into two groups, the physical and the logical. On the physical front, such issues as the various locations and the compatibility of the technology available in the locations must be addressed. These problems are best left to the technologists. The logical issues in many ways are no different to the organisation of face to face meetings, however some issues take on new importance.

\subsection{Physical issues}

\section{Location and time}

The simplest and most important physical issue is that those attending the meeting all understand where they are expected to be on the appointed day, at what time and for how long. In the one organisation this can often be a stumbling block. The video facilities may not be well located and many people may never have used them previously. When stretching across organisations, countries, continents, and time zones this issue is likely to be even more critical. The facilities offered will vary across the following:

Meeting room based voice links, eg. voicepoint.

Individual voice links, eg. telephone bridging equipment.

Meeting room based video links using slow scan technology

Meeting room based video links using high speed communication links, ie television quality.

Individual video links using video 'phones or a similar technology.

\section{Convenience of booking}

The convenience for booking facilities is also an important issue. When the facilities are readily available, sometimes at short notice, then the organisation will make use of them. On the other hand, when the booking of the facilities becomes too difficult for the normal meeting coordinator, the fall back towards face to face meetings can be quick. Within the second year of availability the booking of the video meeting rooms became a formal and bureaucratic exercise. A significant reduction in use by the Faculty of Management could also be identified as the procedures were entrenched. This was particularly the case for ad hoc meetings.

Cost of usage

A similar, but more subtle, pressure is the visibility of the meeting cost. For example, where the cost of technology supported meetings is debited to a visible budget line while the cost of travel and time lost, associated with face to face meetings, is left unconsolidated the use of the facilities will diminish. Even worse, meetings may be cancelled or put on an extended cycle. This results in the saving of "visible" funds while losing coordination and integration impetus. A particularly unproductive result when typically the provision of the facilities involves a significant fixed cost with only a minor marginal cost for usage.

By late 1994 the University had implemented a user pays booking principle for the video conference facilities. The cost structure set aimed to recover the full (capital and expense) investment in video meeting rooms and technology together with a proportion of the capital investment in communications lines. The costs struck were relatively high when compared to travel costs and when compared to the discretional funds available to faculties and the schools within the faculties. In the Faculty of Management this resulted in the cancellation of pilot exercises using the facilities to support teaching across campus to small student cohorts. It also resulted in a further reduction in the use of the facilities for "lower level" meetings, curriculum reviews, unit team discussions etc. Many of these 
meetings were just not held with the result that progress towards across campus integration at the grass roots level was inhibited.

\section{Environment issues}

A number of environment issues are also relevant, for example:

A soundproof room which is local to the workplace is important. The room should be within easy walking distance of most participants' normal offices, and be sufficiently soundproof for confidential conversations.

The size of room should be appropriate for the group, ie a spacious room with comfortable chairs and an appropriately sized table.

A whiteboard link to other meeting locations is often valuable for meetings when wide ranging discussions and problem solving is involved.

The availability of facsimile transmission equipment can often reduce the problems of distributing late material.

Easy access to technical staff can be critical, particularly when participants are not familiar with the technology being used.

The ability to mute the sound transmitted has both positive and negative aspects. By cutting off the sound transmission, people in a local setting can ask for clarification of points missed due to bad transmission etc. But, if people in one location are able to cut off their discussions from the meeting as a whole sub-meetings can break out without the knowledge of even a well organised chair.

\section{Number of participants}

There are also a number of general issues which should be thought about in the context of specific meetings. For most of these there seems to be no clear research to support or guide an answer, for example:

Is there an optimum number of participants?

Is there an optimum number of locations?

Is there a number (participants/locations) above which the technology chosen is not productive?

What other technology can be used to support the meeting?

The question of the number of participants is relevant regardless of the support technology chosen. It does, however, add another dimension to the problem when technology is used to support multi-location meetings.

The question of the number of locations (ie two-way versus multi-way connections) will be answered differently for different meeting types and outcome aims. For example, in the late 1980's many thousands of company employees participated in a world wide hook up for a company announcement from the chairman of Hewlett Packard. A one-way mass communication exercise which swiftly introduced a new corporate structure at the same time to all (or nearly all) employees.

\subsection{Logical issues}

\section{The meeting chair}

To determine just what pre-meeting issues are of importance when planning a remote multi-location meeting the organisers must at the very least:

Establish that a clear meeting chairperson exists

Determine if the meeting chair should lead discussions

Ensure that the meeting chair understands the technology.

Ensure that the chair knows the participants.

It is imperative that a clear meeting chairperson exists and that this chair is recognised by those involved. Without a clear chair a multi-location meeting, even more than a normal face to face meeting, will wander from focal point to focal point and much costly time will be lost. 
The selection and briefing of the meeting chair is guided by a decision on whether the chair should lead most discussions or simply coordinate the discussion. Video format unfortunately lends itself to dominance by the chair. In some contexts this may be acceptable but in many meeting types the chair may need to encourage others to participate. This is, again, a more difficult task when the technology imposes constraints on the participants.

\section{Who drives the technology?}

Ideally the meeting chair should be comfortable in the use of the technology. This is sometimes difficult. Hence, should the need arise, it is imperative that the chair is supported by a "secretariat" well versed in the technology. By ensuring the chair is confident with the technology, the need for extraneous "helpers" can be avoided and the chair can talk others in different locations through any difficulties which might otherwise be used as a "crutch" for lack of involvement.

\section{Know the participants}

The chair should know each of the participants. Again this might seem to be a simplistic observation, but in a technology supported multi-location meeting it is more important for the chair to know the participants, to understand their strengths and weaknesses and, where political negotiations are involved, to be aware of their 'normal' positions on issues to be discussed. Without this knowledge, meetings can be held and decisions reached which are not well accepted by those attending the meeting. Should this occur, the dissenters will often blame the technology for the meeting's outcome when the real culprit is the poorly prepared meeting chair.

\section{Issues impacting those attending}

Having established just who should be responsible for running the meeting, the following questions must be tackled:

Have the meeting participants met each other previously?

Do they know each other's strengths and weaknesses?

Are they familiar with the technology being used?

Has a formal agenda been developed and distributed prior to the meeting?

Has all supporting documentation been distributed prior to the meeting?

Are the meeting participants familiar with the meeting format being used?

If the participants have met each other previously then the business can be commenced immediately. If, however, they are unknown to one another the first meeting should be devoted to gaining this understanding and the amount of business should be limited.

Knowing each other's strengths and weaknesses ensures that those attending the meeting can contribute appropriately. It also enables the chair to ensure that discussion flows smoothly between locations and no single location dominates the discussion.

Familiarity with the technology being used ensures comfort and reduces the times that the technology is seen as a distraction to the achievement of goals.

\section{The use of an agenda}

The distribution of a formal agenda prior to the meeting ensures that all present can contribute at the appropriate stage. Since technology supported multi-location meetings most often have strict time limits, the use of a formal agenda enables the chair to gauge the meeting's progress, to identify issues which may be omitted if time is short, and to obtain quick agreement for any adjustments needed.

The need to distribute all meeting documentation on time is made more critical when meetings are run in a number of remote locations. Distribution and discipline/planning problems which result in "tabled" papers make meetings difficult to chair. It is true that a properly equipped meeting room will also contain a facsimile machine which can be used for last minute document distribution. This is, however, an extremely poor substitute for prior distribution of material. Inevitably the remote location participants develop a feeling of "last minute inclusion" which tends to breakdown the meeting's solidarity. The need for well planned participation may lead to the rejection of the technology rather than the acknowledgment of poor preparation by meeting participants. 


\section{Know the format}

When those attending the meeting are familiar with the meeting format, the business at hand is often quickly dealt with. If, on the other hand, the format of the meeting is new to many attending much time will be lost while people determine just how things will be run. Therefore meeting chairs would be advised to allow additional time, or, preferably, reduce the agenda, in early meetings.

\section{OTHER KEY ISSUES}

\subsection{Reaching consensus in technology supported meetings}

The counting of votes in remote locations can be difficult. Indeed, the notion of meeting quorums for some organisations may need to be addressed. When a decision making board meets with some members in one location and some in another how is the meeting validated if there are insufficient members at any single location to meet the rules? In these cases it may be necessary to amend formal constitutions to allow for a quorum to be calculated from multiple locations.

Technology exists which can simplify the casting of votes. It is rarely implemented however, in simple video and audio meeting arrangements. The chair needs to determine in advance the method to be used should the counting of votes be necessary. Clearly the nature of the outcome sought will influence the approach taken. But, the issue of voting and decisions taken by acclamation must exercise the mind of a well prepared chair.

Group Think issues take on a new meaning when meetings are supported by technology. Some technologies actively encourage Group Think while other technologies inhibit the development of the herd mentality. The ability of remote members of a meeting to convey visual signals to only a subset of the meeting can be an encouragement to this problem.

The ability of people in different locations to break the 'normal' flow of discussion can lead to disjointed discussions and even the dominance of some people who would normally not be so dominant. At times, however, the masking of traditional hierarchical pressures and non-verbal signals may result in freer discussion. The desired meeting outcome should be reflected upon to determine if this is an opportunity or a problem.

\subsection{Security issues}

This is a significant issue which cannot be fully covered in this preliminary overview. People should be made aware of the security issues in the use of technology to support multi-location meetings. In many circumstances security is not a necessary consideration. However, the need for other (technical support style) staff to be involved, the nature of the meeting sites, and the communications lines used may make some meetings vulnerable to security breaches.

\subsection{People issues}

A number of other seemingly minor issues should be given some thought by the meeting organisers and the chair.

The need to start and stop on time becomes more critical with technology supported multi-location meetings. Other groups may have made booking for the technology support rooms. More than a single location is involved in bookings, thus simple time extensions are often difficult. This is a problem for all meeting participants, but one often overlooked by people in organisations with "relaxed" meeting cultures.

Problems will also exist with meetings scheduled over lunch or refreshment breaks. It may be impossible to ensure all meeting members are similarly catered for. 
Problems of people wandering in and out can often be tolerated in face to face meetings. In technology supported multi-location meetings, however, this can be much more difficult for a chair to control and very disconcerting for others attending.

The formality of the meeting, eg first name or formal title basis, will play an important part in the ability of the chair to involve many members. Informal signals are often denied the chair when participants are in other locations. Thus a "random" question to members at other locations should be programmed into the chair's meeting procedures. This can be made easier in meetings run on a less formal basis.

Another problem to be avoided, often associated with tight booking schedules, is where there is a temptation for the meeting to continue at one location after the scheduled meeting ends. If this occurs too frequently it will eventually result in the breakdown of communication between participants and may culminate with people travelling to the central locations simply to ensure all of the meeting is attended.

\section{SUMMARY}

The costs and the benefits of the use of technology to support multi-location meetings are largely in the intangible area. Investments can be justified, however, concentrating on the tangible savings in travel time balancing this with the capital investment and operating costs of technologies to support multi-location meetings. The changes required in corporate culture, to ensure successful integration of the technology into regular decision making, are significant. An understanding of the intangible costs the geographic distribution of an organisation impose is required before this change in corporate culture can be addressed.

\section{FURTHER WORK POSSIBLE}

It would be of use to examine the familiarity those attending have with the technology being used and the impact this has on the perceived satisfaction with the technology for different classes of attendees. Further analysis could be undertaken into the different methods of running multi-location meetings, linking this with the anticipated outcomes and perceived satisfaction with the technology used.

Enough work has been done on Group Decision Support systems for there to be general agreement that, by specially constructing meeting rooms and providing participants with appropriate technology, anonymity can be introduced and this leads to increased participation [DeSanctis \& Gallupe, 1993; Gray \& Nunamaker, 1993]. When the environment of meetings is altered by the use of technology to support meeting members in multiple locations it is possible that participation, by those attending, is altered for reasons of perceived anonymity. This leads to the question:

Under what circumstances does the use of technology increase participation in meetings and where does it limit it?

To establish a set of guidelines for meeting chairs, further analysis into the classification of expected meeting outcomes would be useful. As indicated above, successful technology supported multi-location meetings might well depend upon those attending the meeting as much as the actions of the meeting chair. Some classes of meeting seem to be well translated into multi-location mode, while other classes of meeting seem to fail. This leads to the question:

Can the benefits of multi-location meetings be used to change the corporate culture towards

the more supportable classes of meetings or would this be counter-productive?

The geographical element leading to the choice of Face to Face versus multi-location meetings is but one decision. Equally interesting questions revolve around the time element.

Do meetings need to be synchronous?

The very nature and reason for calling the meeting can also be called into question.

Are there some outcome classes that are better arrived at by taking more time?

Indeed the question addressing "what other technology can be used to support the meeting" raised above could be generalised a little. Computer Mediated Communications (CMC) technology involves one or a combination of the following software: e-mail, bulletin boards, list-servers, shared text data 
bases, etc. The evolution of Groupware or CMC, the most notable product being Lotus Notes, is changing the opportunities open to organisations in the support of group decision making. Group decision making has been previously thought of as the fundamental reason for calling many meetings. Thus the final question to emerge is:

What form of meetings can be eliminated entirely by using new asynchronous group support technology?

\section{REFERENCES}

DeSanctis, G., \& Gallupe, B. (1993). Group Decision Support Systems: A New Frontier. In S. R. H. \& W. H. J. (Eds.), Decision Support Systems New Jersey: Prentice-Hall International.

Gray, P., \& Nunamaker, J. F. (1993). Group Decision Support Systems. In S. R. H. \& W. H. J. (Eds.), Decision Support Systems New Jersey: Prentice-Hall International.

Hewett, W. G., \& Symons, I. (1992). A TCF Organisation In a Changing Environment: Some Analysis For The Writing Of An Australian Case Study. In The Australian and New Zealand Academy of Management Conference, December 6-9 1992., . Sydney:

Renton, N. E. (1994). Guide for Meetings and Organisations Volume 2 Meetings. Sydney: The Law Book Company Limited.

Shockley-Zalabak, P. (1991). Fundamentals of Organizational Communication Second Edition. White Plains: Longman Publishing Group.

\section{BIOGRAPHY}

Bill Hewett has been actively working in the IT industry for over 30 years, Bill has worked in both IT consulting/service organisations and companies applying IT. Ten of these years were spent as an executive in a medium sized company responsible to the board for the delivery of IT services.

Since joining Deakin University in 1991 Bill has maintained his involvement with commercial applications of IT by acting as a consultant specialising in the evaluation of IT services. Bill's research interests lie in this area, in particular in attempting to define an appropriate role for IT in organisations, and examining the effectiveness of the IT function. 